\title{
Exogenous IGF-1 improves tau pathology and neuronal pyroptosis in high-fat diet mice with cognitive impairment
}

\section{Guanghong Sui}

Tianjin Anding Hospital

\section{Lu Wang}

Tianjin Medical University General Hospital

\section{Caixia Yang}

Tianjin Anding Hospital

\section{Mengtian Guo}

Tianjin Medical University General Hospital

\section{Xiangyang Xiong}

Tianjin Medical University General Hospital

\section{Zheng Chen}

Tianjin Anding Hospital

Feng Wang ( $\nabla$ wangfeng0921@126.com )

Tianjin Medical University General Hospital

\section{Research Article}

Keywords: Cognitive impairment, Insulin-like growth factor I, Obesity, Pyroptosis, Tau proteins

Posted Date: February 11th, 2021

DOl: https://doi.org/10.21203/rs.3.rs-158607/v1

License: (c) (i) This work is licensed under a Creative Commons Attribution 4.0 International License. Read Full License

Version of Record: A version of this preprint was published at Metabolic Brain Disease on July 16th, 2021. See the published version at https://doi.org/10.1007/s11011-021-00787-4. 


\section{Abstract}

\section{Background}

Insulin-like growth factor-1 (IGF-1) improves obesity-induced cognitive impairment, but its mechanism is not fully clarified. The aim of the study was to reveal whether IGF-1 treated cognitive impairment by improving tau pathology and neuronal pyroptosis in high-fat diet mice, and to further explore its molecular mechanisms involved.

\section{Methods}

During in vitro experiment, C57BL/6J mice were fed with high-fat diet, and were treated with PEG-IGF-1, IGF-1 receptor blocker AXL1717, HO-1 blocker Znpp IX or their combinations. Cognitive function was evaluated using Morris water maze. Expression of Nrf-2, HO-1, p-tau, NLRP3, Caspase-1 and IL-1 $\beta$ in hippocampus was determined using western blotting. Pyroptosis rate in hippocampus was measured using flow cytometry. During in vivo experiment, HN-h cells were treated with palmic acid, pyroptosis blocker nonecrosulfonamide or their combinations. The expression of the proteins and pyroptosis rate were also measured using western blotting and flow cytometry.

\section{Results}

During in vitro experiment, high-fat diet mice showed cognitive impairment, hyperphospharylation of tau protein and significant neuronal pyroptosis in hippocampus compared with the sham mice. After exogenous IGF-1 treatment, these abnormalities were reversed, and Nrf-2/HO-1 signaling pathway was activated. Inhibition of such signaling pathway using IGF-1 receptor blocker or HO-1 blocker redeteriorated cognitive function, neuronal pyroptosis and tau pathology in hippocampus. During in vivo experiment, inhibition of pyroptosis using its blocker improved tau pathology in palmic acid-treated HN-h cells.

\section{Conclusion}

Exogenous IGF-1 improved cognitive impairment, tau pathology and neuronal pyroptosis induced by highfat diet through activation of $\mathrm{Nrf}-2 / \mathrm{HO}-1$ signaling pathway.

\section{Introduction}

Nowadays, obesity is a common condition all around the world. In the United States, nearly $40 \%$ of the population is affected by obesity, and more than two-third of the people are overweight (Hansen et al. 2020). In China, from 1992 to 2015, obesity rate elevates from 3-12\%, and overweight rate rises from 13-30\% (Zhang et al. 2020). For each obese patient, obesity decreases athletic ability, and more importantly, it causes or aggravates chronic diseases. Compared with cardiovascular diseases and diabetes mellitus, cognitive impairment, as one kind of chronic disease, is very easy to be ignored in 
obese patients, but is very harmful to health (Wang et al. 2017; Zhang et al. 2020). So, it is meaningful to fully explore the pathogenesis and therapy of the obesity-induced cognitive impairment.

There are a large number of pathophysiological disorders in human bodies relating to cognitive impairment. In Alzheimer's disease, neurofibrillary tangle with neurotoxicity is thought to be characteristic of the pathology, and contributes to the cognitive impairment (Abu-Rumeileh et al. 2018). Neurofibrillary tangle is formed by an abnormal tangle of tau protein, and the latter is also discovered in mouse models of obesity and metabolic disorders (Špolcová et al. 2015; Platt et al. 2016). Therefore, we have reason to believe that tau protein is also involved in the development of cognitive impairment in obesity.

Pyroptosis is a programmed cell death process, and pyroptosis in neurons is associated with neuroinflammation. Previous studies suggest that neuroinflammation develops in brain tissue of obese patients (Miller and Spencer 2014), and other studies report that neuronal pyroptosis causes or aggravates several types of cognitive impairment (Yin et al. 2018; Fu et al. 2019). So, we speculate that obesity might contribute to neuronal pyroptosis, and further deteriorate cognitive function.

Insulin-like growth factor-1 (IGF-1) is a widely distributed polypeptide in human bodies, and plays a protective role in cognitive impairment (Farias Quipildor et al. 2019; Yang et al. 2019). Our study reports that exogenous IGF-1 improves cognitive function in high-fat diet mice, and inhibits inflammation and oxidative stress in hippocampus tissue (Wang et al. 2020). Nrf2/HO-1 is an important antioxidant stress signaling pathway (Ali et al. 2018), and is also involved in anti-inflammatory process in nervous system (Luo et al. 2017; Ma et al. 2020). Previous studies explore the relationship between IGF-1 and Nrf2/HO-1 signaling pathway, and report that IGF-1 is able to activate Nrf2, and might play a regulatory role in Nrf2/HO-1 signaling pathway (Riis et al. 2020; Wang et al. 2017).

Taken together, the present study tried to reveal whether exogenous IGF-1 improved tau pathology and neuronal pyroptosis through activation of $\mathrm{Nrf} 2 / \mathrm{HO}-1$ signaling pathway in a high-fat diet (HFD) mouse model with cognitive impairment. After that, we conducted an in vitro test to explore the relationship between tau pathology and neuronal pyroptosis in a high-fat cell model.

\section{Materials And Methods}

The study was approved by the ethics committees of Tianjin Medical University General Hospital and Tianjin Anding Hospital.

\subsection{Grouping, modeling and intervention in mice}

Fifty male C57BL/6J mice (four weeks old) were purchased from laboratory animal center of the academy of military medical sciences (Beijing, China). The mice were supplied with normal, balanced and adequate diets, and were kept at room temperature with a light/dark cycle $(12 \mathrm{~h} / 12 \mathrm{~h})$ for seven days before our experiments. 
Then, the animals were divided into five groups according to randomization principle: sham group, HFD group, IGF-1 treatment group (IGF group), IGF-1 receptor inhibition group (IGFR_I group) and HO-1 inhibition group (HO_I group). Each group contained 10 mice.

After grouping, the mice in the HFD, IGF, IGFR_I and HO_I groups were treated with HFD (D12492, Research Diets) for 12 weeks. Its energy composition was fats (60\%), carbohydrates (20\%) and proteins (20\%). During the same period (12 weeks), the sham mice were fed with normal diet (D12450B, Research Diets). Their body weights were measured weekly, and their fasting serum glucose and lipids were determined at the end of the modeling process. Briefly, fasting blood was obtained from caudal vein of each mouse, and an automatic biochemical analyzer (Hitachi 7170, Hitachi, Japan) was adopted to measure these biochemical markers.

In the next step, the mice in the IGF, IGFR_I and HO_I groups were treated with polyethylene glycol - IGF-1 (PEG-IGF-1) by intraperitoneal injection for four weeks (1 mg/kg, twice a week) (Sama et al. 2018). Meanwhile, the mice in the IGFR_I and HO_I groups were separately treated with IGF-1 receptor blocker AXL1717 and HO-1 blocker Znpp IX by intraperitoneal injection for four weeks (AXL1717: $20 \mathrm{mg} / \mathrm{kg}$, once a day; Znpp IX: 20 mg/kg, twice a week) (Wang et al. 2018; Xu et al. 2019). The mice in the sham group were treated with $0.9 \%$ normal saline by intraperitoneal injection once a day for four weeks. AXL1717 and Znpp IX were separately obtained from GlpBio and Sigma-Aldrich (USA), and were dissolved and diluted using DMSO according to their instructions. In this period, the sham mice were also fed with normal diet, and the other mice were fed with HFD.

\subsection{Grouping, modeling and intervention in cells}

A human neurons-hippocampal $(\mathrm{HN}-\mathrm{h})$ cell line was purchased from ScienCell Research Laboratories (USA), and was cultured according to routine procedure. Briefly, the cells were maintained in poly-L-lysinecoated cell culture flasks with specific neuronal medium (NM, Cat. \#1521). The flasks were placed at $37^{\circ} \mathrm{C}$ in an atmosphere containing $5 \%$ carbon dioxide. The medium was renewed every 48 hours. With the division and growth of the cells, they were transferred to new flasks for more space. Eventually, the cells were seeded into 6-well plates for our experiments.

The prepared cells were divided into control group, palmic acid treatment group (PA group) and pyroptosis inhibition group (Pyr_l group). Each group contained ten wells. The cells in the PA and Pyr_l groups were treated with one kind of saturated fatty acids - palmic acid $(800 \mu \mathrm{mol} / \mathrm{l})$ for 12 hours, and the cells in the Pyr_I group were also treated with pyroptosis blocker necrosulfonamide $(4 \mu \mathrm{mol} / \mathrm{l})$ for 12 hours (Yao et al. 2018). Palmic acid and necrosulfonamide were purchased from Sigma-Aldrich (USA). Palmic acid was dissolved using $\mathrm{NaOH}(0.1 \mathrm{~mol} / \mathrm{l})$ at $70^{\circ} \mathrm{C}$ for 5 minutes and then mixed using bovine serum albumin $(10 \%)$ at $55^{\circ} \mathrm{C}$ for 10 minutes. The mixture was added with specific neuronal medium (NM, Cat. \#1521) to form the concentration of $800 \mu \mathrm{mol} / \mathrm{l}$. Necrosulfonamide was dissolved and configured by DMSO $(0.1 \%)$ to produce the concentration of $4 \mu \mathrm{mol} / \mathrm{l}$.

\subsection{Cognitive function test in mice}


After modeling and intervention, cognitive function was evaluated using Morris water maze. Detailed steps were described in our previous literature (Barnhart et al. 2015). During a day of adaptive training, the rats were arranged to familiarize themselves with an experimental pool with a platform in it.

Subsequently, navigation test was conducted on day 2 to day 5 . The rats were randomly placed any quadrant in the pool, and their trajectories and times were recorded using a video-tracking system (TSE Systems, Bad Homburg, Germany). "Escape latency" was defined as the length of time a rat took from being placed in a pool to climbing the platform. If the escape latency of one rat was longer than 60 seconds, its escape latency was recorded as 60 seconds. This rat were also guided to the platform and placed on it for another 30 seconds. After the navigation test, the platform was removed in order to perform spatial probe test on Day 6. Each rat was placed in the pool again, and let them swim in the pool for 120 seconds. The length of time each rat spent in the target quadrant and the number of times it went through the target quadrant were recorded. The "target quadrant" was defined as the quadrant where the platform was originally located.

\subsection{Specimen collection and preparation in mice}

After the behavioral evaluation, the mice were humanely euthanized under anesthesia. Cerebrospinal fluid specimen was obtained from cisterna magna using a glass capillary tube (Liu and Duff 2008). Hippocampus tissue was collected and cleaned using double distilled water. The fresh tissue was cut as small as possible and digested with trypsin (0.2区) for about 30 minutes until the tissue was dispersed. The tissue suspension was filtered with a 100 mesh nylon filter. The obtained cell suspension was centrifugated at $1000 \mathrm{rpm}$ for 5 minutes, and was mixed with PBS to form a hippocampus neuron suspension for further experiments.

\subsection{Levels of tau proteins in cerebrospinal fluids}

Cerebrospinal fluid levels of p-tau (Ser 181) and p-tau (Thr 205) were determined using a Singulex Erenna immunoassay platform (USA) (Hastings et al. 2017). HT7 was adopted as capture antibody. AT270 and AT8 which separately recognized p-tau (Ser 181) and p-tau (Thr 205) served as detection antibodies. In the process, the measurement was performed in the presence of HaltR Protease and Phosphatase Inhibitor Cocktail (Thermo Fisher). Briefly, in 96-well plate, each well contained $10 \mathrm{mg}$ capture beads, $50 \mu$ S Singulex assay buffer and $50 \mu$ l cerebrospinal fluids (or standard peptide), which was diluted in PBS-T $\left(0.2 \%\right.$ Tween 20 in PBS). The plate was incubated at $25^{\circ} \mathrm{C}$ for 4 hours, and was washed using a Biotek $405^{\text {TM }}$ TS microplate washer. Then, $20 \mu \mathrm{l}$ AT270 or AT8 antibody $(50 \mathrm{ng} / \mathrm{ml})$ was added into the plate, and was incubated and shaken overnight at $25^{\circ} \mathrm{C}$. The cerebrospinal fluid specimen was developed according to the instruction, and the result was analyzed using Sgx link software (Singulex Erenna).

\subsection{Protein expression in hippocampus tissue and HN-h cells}

Expression of Nrf2, HO-1, p-tau (Ser 181), p-tau (Thr 205), t-tau, NLRP3, cleaved Caspase-1, IL-1 $\beta$ and GAPDH in the hippocampus tissue and HN-h cells were determined using western blotting. 
Briefly, RIPA lysis buffer (Thermo Fisher Scientific, MA, USA) was adopted to extract the protein from the specimens, and Pierce ${ }^{\mathrm{TM}}$ modified Lowry protein assay kit (Thermo Fisher Scientific, MA, USA) was used to measure the total protein levels. A certain amount of protein $(50 \mu \mathrm{g})$ was separated using $12 \%$ sodium dodecyl sulfate-polyacrylamide gel electrophoresis, and then were transferred onto a Bio-Rad's nitrocellulose membrane (Hercules, CA, USA). After 2-hour blocking with $5 \%$ skimmed milk at $25^{\circ} \mathrm{C}$, the membrane was incubated anti-Nrf2 (Ser 9) (CST, \#12721, 1:1000 dilution), anti-HO-1 (CST, \#82206, 1:1000 dilution), anti-p-tau (Ser 181) (CST, \#12885, 1:1000 dilution), anti-p-tau (Thr 205) (CST, \#49561, 1:1000 dilution), anti-t-tau (CST, \#46687, 1:1000 dilution), NLRP3 (CST, \#15101, 1:1000 dilution), anticleaved Caspase-1 (CST, \#89332, 1:1000 dilution), IL-1 $\beta$ (CST, \#31202, 1:1000 dilution) and anti-GAPDH (CST, \#5174, 1:1000 dilution) at $4^{\circ} \mathrm{C}$ overnight. Subsequently, the membrane was incubated with an antirabbit IgG, HRP-linked secondary antibody (\#7074, CST). Chemiluminescence detection was adopted to visualize the immunoreactive bands (Immolilon Western, USA). Image $\mathrm{J}$ software was used to measure the intensities of the bands.

\subsection{Pyroptosis rate in hippocampus tissue and HN-h cells}

Pyroptosis rate in the specimens was determined using a FAM-FLICA Caspase-1 detection kit (ImmunoChemistry) (Zeng et al. 2019). Briefly, prepared cells were stained with FAM-FLICA (10 $\mu \mathrm{l}$ ) and PI $(5 \mu \mathrm{l})$ at $26^{\circ} \mathrm{C}$ for 20 minutes. Then, fluorescence intensities of the specimens were measured by a Coulter Epics XL flow cytometer. Pyroptosis rate was calculated using a formula: number of doublepositive cells / number of total cells $\times 100 \%$.

\subsection{Statistical analysis}

Continuous variable in the study was expressed using mean \pm standard deviation. Variable comparison between two groups was conducted using independent sample $t$ test. Variable comparison among more than two groups was performed using variance analysis (ANOVA) with LSD test. P value less than 0.05 was regarded as statistically significant. All statistical analysis was performed using Statistical Product and Service Solutions (SPSS) version 19.0.

\section{Results}

\subsection{Metabolic disorders in mice}

As shown in Figure 1, levels of body weight, fasting glucose, total cholesterol and triglycerides were higher in the HFD group than in the normal diet group $(P \otimes 0.001, P=0.001, P \otimes 0.001, P \unrhd 0.001$, respectively). These findings indicated that long-term HFD caused significant metabolic disorders in the mice.

\subsection{Cognitive function in mice}

As shown in Figure 2, compared with the sham group, escape latency was higher and the other two markers (i.e. percentage of time spent in target quadrant and number of times crossing the platform area) 
was lower in the HFD group ( $\mathrm{P} \otimes 0.001, \mathrm{P} \otimes 0.001, \mathrm{P} \otimes 0.001$, respectively). PEG-IGF-1 treatment reversed the abnormalities in the IGF group ( $P \otimes 0.001, P \otimes 0.001, P \otimes 0.001$, respectively). Both IGF-1 receptor blocker and $\mathrm{HO}-1$ blocker deteriorated the escape latency, percentage of time spent in target quadrant and number of times crossing the platform area again in the IGFR_I and HO_I groups (IGFR_I: P $\otimes 0.001$, $P \otimes 0.001, P=0.005$, respectively; HO_I: $P \otimes 0.001, P \otimes 0.001, P=0.004$, respectively). The findings indicated that IGF-1 improved HFD-induced cognitive impairment involving the activation of IGF-1 receptor and $\mathrm{HO}-1$.

\subsection{Activation of Nrf2/HO-1 signaling pathway in hippocampus tissue}

In Figure 3, compared with the sham group, the expression of Nrf2 and $\mathrm{HO}-1$ were significantly decreased in the HFD group (P凶0.001, P $\otimes 0.001$, respectively). And, PEG-IGF-1 elevated the expression of these two proteins $(P=0.006, P \otimes 0.001$, respectively). Furthermore, IGF-1 receptor blocker inhibited the expression of Nrf2 and HO-1 in the IGFR_I group $(P=0.026, P \otimes 0.001$, respectively), while HO-1 blocker only inhibited the expression of HO-1 in the HO_I group ( $\mathrm{P} \otimes 0.001)$. These findings indicated that HFD caused the down-regulation of Nrf2/HO-1 signaling pathway, which can be alleviated by IGF-1 treatment.

\subsection{Pyroptosis in hippocampus tissue}

In Figure 4A - 4D, compared with the sham group, the expression of NLRP3, Caspase-1 and IL-1 $\beta$ were upregulated in the HFD group ( $\mathrm{P} \otimes 0.001, \mathrm{P} \otimes 0.001, \mathrm{P} \otimes 0.001$, respectively). In the IGF group, PEG-IGF-1 inhibited the expression of these three proteins $(P \otimes 0.001, P=0.001, P=0.005$, respectively). Both IGF-1 receptor blocker and HO-1 blocker in the IGFR_I and HO_I groups up-regulated the expression of NLRP3, caspase-1 and IL-1 $\beta$ compared with the IGF group (IGFR_I: $P=0.008, P=0.007, P=0.016$, respectively; HO_I: $P=0.003, P=0.001, P=0.021$, respectively).

In Figure 4E - 4F, pyroptosis rate was increased in the HFD group than in the sham group (P区0.001). PEGIGF-1 treatment in the IGF group decreased the rate compared with the HFD group $(P=0.007)$.

Furthermore, the rate was re-increased in the IGFR_I and HO_I groups $(P=0.025, P=0.019$, respectively). These findings indicated that IGF-1 inhibited the pyroptosis in the hippocampus tissue through the activation of Nrf2/HO-1 signaling pathway.

\subsection{Expression of tau protein in mice}

In Figure 5A - 5B, compared with the sham group, cerebrospinal fluid levels of $p$-tau (Ser 181) and p-tau (Thr 205) were increased in the HFD group (P凶0.001, $P \otimes 0.001$, respectively). In the IGF group, PEG-IGF-1 treatment decreased the levels of these two proteins $(P=0.023, P=0.012$, respectively). In the IGFR_I and HO_I groups, IGF-1 receptor blocker and HO-1 blocker again elevated the levels of p-tau (Ser 181) and ptau (Thr 205) in cerebrospinal fluids (IGFR_I: $P=0.036, P=0.013$, respectively; $H O \_I: P=0.010, P=0.002$, respectively).

In Figure 5C - 5E, the expression of p-tau (Ser 181) and p-tau (Thr 205) were higher in the HFD group than in the sham group ( $P \otimes 0.001, P \otimes 0.001$, respectively). And, PEG-IGF-1 treatment significantly 
inhibited the expression of them in the IGF group $(P=0.039, P=0.015$, respectively). The blockers mentioned above weakened the effect of PEG-IGF-1 on the expression of p-tau (Ser 181) and p-tau (Thr 205) (IGFR_I: $P=0.040, P=0.018$, respectively; $H O \_I: P=0.042, P=0.005$, respectively). These findings indicated that IGF-1 reversed tau pathology caused by HFD through the activation of Nrf2/HO-1 signaling pathway.

\subsection{Pyroptosis in HN-h cells}

In Figure 6, expression of three pyroptosis-related proteins (i.e. NLRP3, Caspase-1 and IL-1 $\beta$ ) and pyroptosis rate were higher in the PA group than in the control group $(P \otimes 0.001, P \otimes 0.001, P \otimes 0.001$, $P \otimes 0.001$, respectively). Pyroptosis blocker necrosulfonamide in the Pyr_l group significantly decreased IL-1 $\beta$ expression and pyroptosis rate compared with the PA group $(P=0.009, P=0.001$, respectively), but can not affect the expression of NLRP3 and Caspase-1 $(P=0.483, P=0.483$, respectively). These findings indicated that necrosulfonamide alleviated the pyroptosis which was induced by palmic acid in HN-h cells.

\subsection{Expression of tau protein in HN-h cells}

In Figure 7, expression of p-tau (Ser 181) and p-tau (Thr 205) were higher in the PA group than in the control group ( $P \otimes 0.001, P \otimes 0.001$, respectively). Necrosulfonamide in the Pyr_I group significantly inhibited the expression of $p$-tau (Ser 181) and p-tau (Thr 205) compared with the PA group ( $P=0.022, P$ $=0.013$, respectively). These findings indicated that pyroptosis contributed to the development of tau pathology in HN-h cells.

\section{Discussion}

Obesity is a common health problem all around the world. It is well known that obese patients suffer from sub-clinical inflammation in whole bodies including brain tissue (Miller and Spencer 2014). Furthermore, inflammation is closely related to pyroptosis (one kind of inflammatory process of death), and is also related to many other pathophysiological processes, such as apoptosis, oxidative stress and mitochondrial dysfunction. All of the disorders contribute to the development of cognitive impairment (Miller and Spencer 2014). So, it is of great significance to explore the role of inflammation in obesityrelated cognitive diseases.

In the study, we adopted long-term HFD to induce a mouse model of obesity. The reason why we chose HFD to do the modeling was that the strategy was much closer to the reality than surgery, genetic modification and other modeling methods, which may be helpful for the reliability of the conclusion (Heydemann 2016). During the modeling process, we routinely monitored the body weights of the mice, and also measured several serological indicators (fasting blood sugar, lipids and IL-1 $\beta$ ). All the results indicated that HFD successfully induced obesity, metabolic disorder and inflammation in the mice. 
Then, the study adopted PEG-IGF-1, but not free IGF-1 to treat HFD mice. PEG extends half-life of the regent and reduces the number of injection, which helps ensure efficacy and reduces animal suffering. We found that exogenous IGF-1 significantly improved cognitive impairment, neuronal pyroptosis and tau pathology in HFD mice, which was consistent with our previous study (Wang et al. 2020). In that study, we reported that IGF-1 alleviated cognitive impairment, inflammation and oxidative stress induced by HFD partly through PI3K/Akt/CREB signaling pathway.

The fact in the study also proved that exogenous IGF-1 played its biological role by activation of Nrf2/HO-1 signaling pathway. The latter is an antioxidant stress signaling pathway, and is also involved in the regulation of inflammation, apoptosis and energy metabolism (Luo et al. 2017; Ali et al. 2018; Ma et al. 2020). Our study added some updated knowledge about the regulative effect of the signaling pathway on cognitive function, neuronal pyroptosis and tau pathology in HFD models.

As mentioned above, hyperphospharylation of tau protein is related to the development of Alzheimer's disease (Zhang et al. 2020). Our present study also found tau pathology in hippocampus of HFD mice, which gave us a hint that tau protein might contribute to HFD-induced cognitive impairment. Furthermore, potential relationship between neuronal pyroptosis and tau pathology was explored in the study. We used a HN-h cell line which was treated with palmic acid to establish a high-fat in vitro model. After inhibition of pyroptosis using its blocker, tau pathology was remarkably alleviated in $\mathrm{HN}$-h cells. These facts proved that pyroptosis, as one kind of inflammatory death, might stimulate the production of tau protein or weaken its removal. Improving pyroptosis may has a certain therapeutic implication for tau pathology. Meanwhile, we again verified the important role of inflammation in tau pathology.

In conclusion, exogenous IGF-1 improved cognitive impairment, hippocampal tau pathology and neuronal pyroptosis induced by HFD through activation of Nrf-2/HO-1 signaling pathway in mice. Furthermore, we provided some interesting evidences that neuronal pyroptosis may be an potential promoter of tau pathology.

\section{Declarations}

Funding: Not applicable.

Conflicts of interest: Not applicable.

\section{Availability of data and material:}

The data can not be shared because this is an ongoing study.

\section{Code availability:}

All analysis is performed by Statistical Product and Service Solutions 19.0.

\section{Authors' contributions:}


Sui Guanghong, Wang Lu, Chen Zheng and Wang Feng conduct study design, literature research and manuscript preparation. Yang Caixia, Guo Mengtian and Xiong Xiangyang carry out experiment and performed data analysis.

\section{Ethics approval:}

The study is approved by the ethics committees of Tianjin Medical University General Hospital and Tianjin Anding Hospital.

\section{Consent to participate:}

This is an in vivo and in vitro study, and no human subjects are included.

\section{Consent for publication:}

All authors agree to submit this article to your journal.

\section{Acknowledgments:}

We want to thank Prof. Jia Qifen from TIANJIN UNIVERSITY (China) for the funding and laboratory support for this project.

\section{References}

1. Abu-Rumeileh S, Capellari S, Parchi P (2018) Rapidly Progressive Alzheimer's Disease: Contributions to Clinical-Pathological Definition and Diagnosis. J Alzheimers Dis 63:887-897

2. Ali T, Kim T, Rehman SU et al (2018) Natural Dietary Supplementation of Anthocyanins via $\mathrm{PI3K} /$ Akt/Nrf2/HO-1 Pathways Mitigate Oxidative Stress, Neurodegeneration, and Memory Impairment in a Mouse Model of Alzheimer's Disease. Mol Neurobiol 55:6076-6093

3. Barnhart CD, Yang D, Lein PJ (2015) Using the Morris water maze to assess spatial learning and memory in weanling mice. PLoS One 10:e0124521

4. Farias Quipildor GE, Mao K, Hu Z et al (2019) Central IGF-1 protects against features of cognitive and sensorimotor decline with aging in male mice. Geroscience 41:185-208

5. Fu Q, Wu J, Zhou XY et al (2019) NLRP3/Caspase-1 Pathway-Induced Pyroptosis Mediated Cognitive Deficits in a Mouse Model of Sepsis-Associated Encephalopathy. Inflammation 42:306318

6. Hansen AR, Rustin C, Opoku ST et al (2020) Trends in US adults with overweight and obesity reporting being notified by doctors about body weight status, 1999-2016. Nutr Metab Cardiovasc Dis 30:608-615

7. Hastings NB, Wang X, Song L et al (2017) Inhibition of O-GlcNAcase leads to elevation of O-GlcNAc tau and reduction of tauopathy and cerebrospinal fluid tau in rTg4510 mice. Mol Neurodegener 12:39 
8. Heydemann A (2016) An Overview of Murine High Fat Diet as a Model for Type 2 Diabetes Mellitus. J Diabetes Res 2016:2902351

9. Liu L, Duff K (2008) A Technique for Serial Collection of Cerebrospinal Fluid From the Cisterna Magna in Mouse. J Vis Exp :960

10. Luo D, Guo Y, Cheng Y et al (2017) Natural product celastrol suppressed macrophage M1 polarization against inflammation in diet-induced obese mice via regulating $\mathrm{Nrf} 2 / \mathrm{HO}-1, \mathrm{MAP}$ kinase and NF-kappaB pathways. Aging 9:2069-2082

11. Ma Z, Lu Y, Yang F et al (2020) Rosmarinic acid exerts a neuroprotective effect on spinal cord injury by suppressing oxidative stress and inflammation via modulating the Nrf2/HO-1 and TLR4/NFkappaB pathways. Toxicol Appl Pharmacol 397:115014

12. Miller AA, Spencer SJ (2014) Obesity and neuroinflammation: a pathway to cognitive impairment. Brain Behav Immun 42:10-21

13. Platt TL, Beckett TL, Kohler K et al (2016) Obesity, diabetes, and leptin resistance promote tau pathology in a mouse model of disease. Neuroscience 315:162-174

14. Riis S, Murray JB, O'Connor R (2020) IGF-1 Signalling Regulates Mitochondria Dynamics and Turnover through a Conserved GSK-3beta-Nrf2-BNIP3 Pathway. Cells 9:147

15. Sama DM, Carlson SW, Joseph B et al (2018) Assessment of systemic administration of PEGylated IGF-1 in a mouse model of traumatic brain injury. Restor Neurol Neurosci 36:559-569

16. Špolcová A, Mikulášková B, Holubová M et al (2015) Anorexigenic lipopeptides ameliorate central insulin signaling and attenuate tau phosphorylation in hippocampi of mice with monosodium glutamate-induced obesity. J Alzheimers Dis 45:823-835

17. Wang F, Wang L, Wang $Y$ et al (2020) Exogenous IGF-1 improves cognitive function in rats with highfat diet consumption. J Mol Endocrinol 64:115-123

18. Wang F, Zhao M, Han Z et al (2017) Association of body mass index with amnestic and nonamnestic mild cognitive impairment risk in elderly. BMC Psychiatry 17:334

19. Wang $\mathrm{H}$, Zhang $\mathrm{H}, \mathrm{Cao} \mathrm{F}$ et al (2018) Protection of insulinlike growth factor 1 on experimental peripheral neuropathy in diabetic mice. Mol Med Rep 18:4577-4586

20. Wang Z, Xiong L, Wang G et al (2017) Insulin-like growth factor-1 protects SH-SY5Y cells against beta-amyloid-induced apoptosis via the PI3K/Akt-Nrf2 pathway. Exp Gerontol 87:23-32

21. Xu J, Lei S, Ye G (2019) Dexmedetomidine attenuates oxidative/nitrative stress in lung tissues of septic mice partly via activating heme oxygenase-1. Exp Ther Med 18:3071-3077

22. Yang Y, Liang S, Li Y et al (2019) Effects of early administration of insulin-like growth factor-1 on cognitive function in septic encephalopathy. Neuropsychiatr Dis Treat 15:323-337

23. Yao H, Tao X, Xu L et al (2018) Dioscin alleviates non-alcoholic fatty liver disease through adjusting lipid metabolism via SIRT1/AMPK signaling pathway. Pharmacol Res 131:51-60

24. Yin L, Bao F, Wu J et al (2018) NLRP3 inflammasome-dependent pyroptosis is proposed to be involved in the mechanism of age-dependent isoflurane-induced cognitive impairment. $\mathrm{J}$ 
Neuroinflammation 15:266

25. Zeng R, Luo D, Li H et al (2019) MicroRNA-135b alleviates MPP +-mediated Parkinson's disease in in vitro model through suppressing Fox01-induced NLRP3 inflammasome and pyroptosis. J Clin Neurosci 65:125-133

26. Zhang L, Wang Z, Wang X et al (2020) Prevalence of overweight and obesity in China: Results from a cross-sectional study of 441 thousand adults, 2012-2015. Obes Res Clin Pract 14:119-126

27. Zhang S, Zhao M, Wang F et al (2020) Relationship between normal weight obesity and mild cognitive impairment is reflected in cognitive-related genes in human peripheral blood mononuclear cells. Psychogeriatrics 20:35-43

\section{Figures}
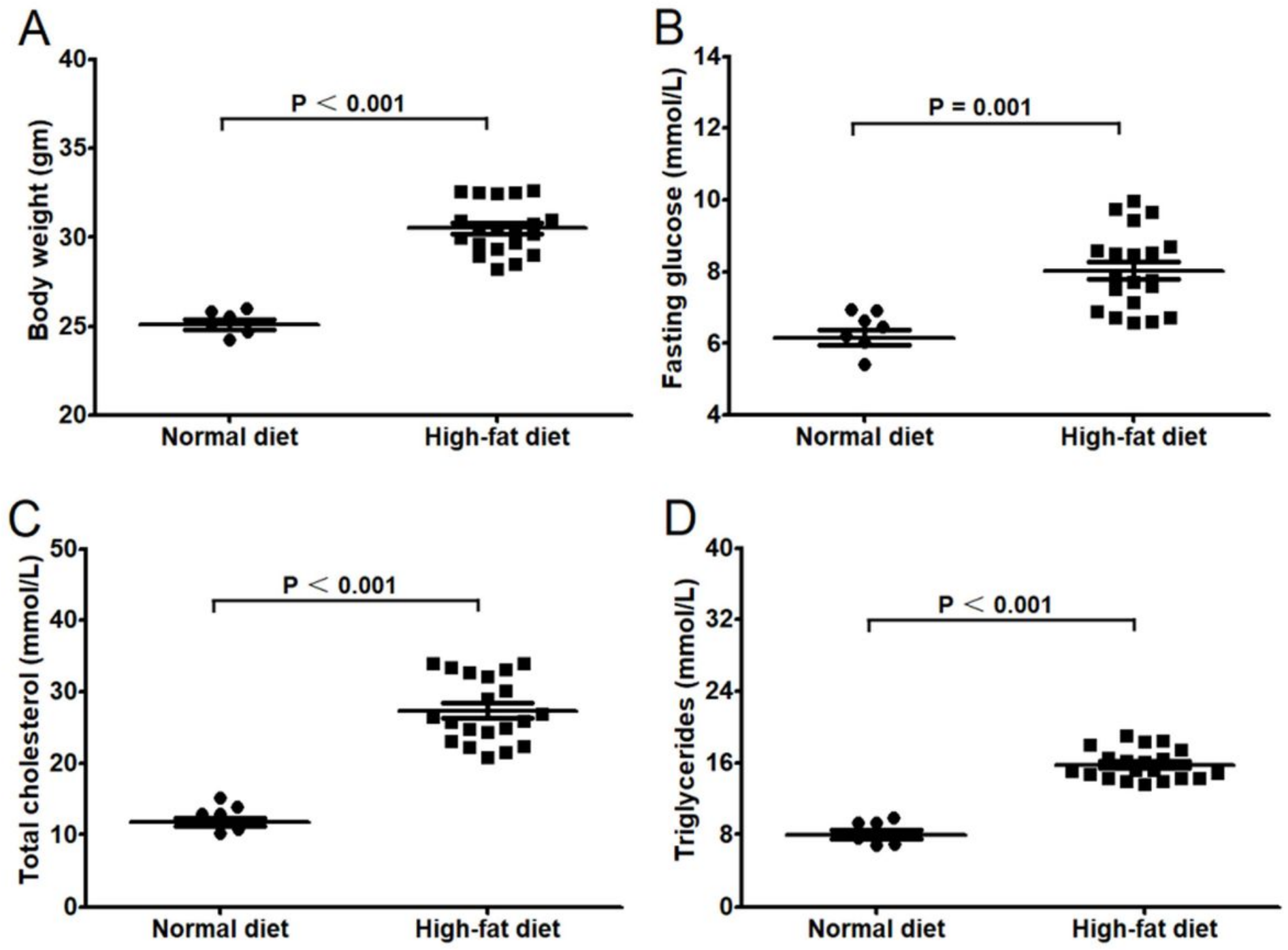

Figure 1

Several metabolic markers in normal and high-fat diet mice Fifty mice were divided into five groups, and each groups had ten mice. Among them, the mice in one group served as controls and were fed with 
normal diet. The mice in the other four groups were fed with high-fat diets. Levels of several metabolic markers were compared between the normal diet rats $(n=10)$ and high-fat diet rats $(n=40)$. Variable comparison between different groups was performed using independent sample t test. " $\mathrm{P} \otimes 0.05$ " was regarded as statistically significant.
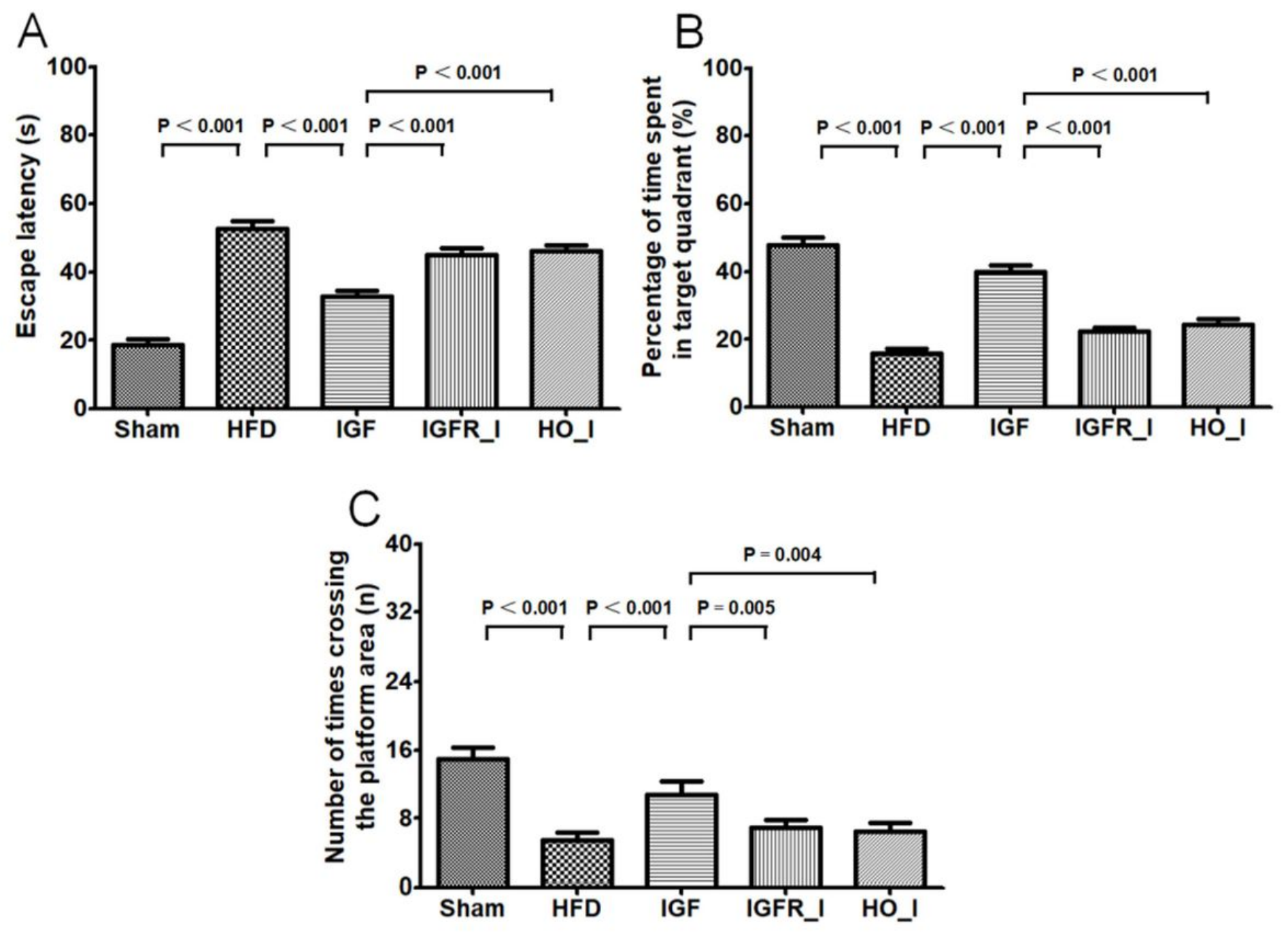

\section{Figure 2}

Cognitive function in animal groups The mice were randomly divided into five groups: sham group, HFD group, IGF-1 treatment group (IGF group), IGF-1 receptor inhibition group (IGFR_I group) and HO-1 inhibition group (HO_I group). Each group contained ten mice. Except the sham mice, all the mice were fed with high-fat diet. Then, the mice in the IGF, IGFR_I and HO_I groups were treated with polyethylene glycol - IGF-1, and the mice in the IGFR_I and HO_I groups were separately treated with IGF-1 receptor blocker AXL1717 and HO-1 blocker Znpp IX. Cognitive function was assessed using Morris water maze. Variable comparison between different groups was performed using variance analysis (ANOVA) with LSD test. $P \otimes 0.05$ was regarded as statistically significant. 

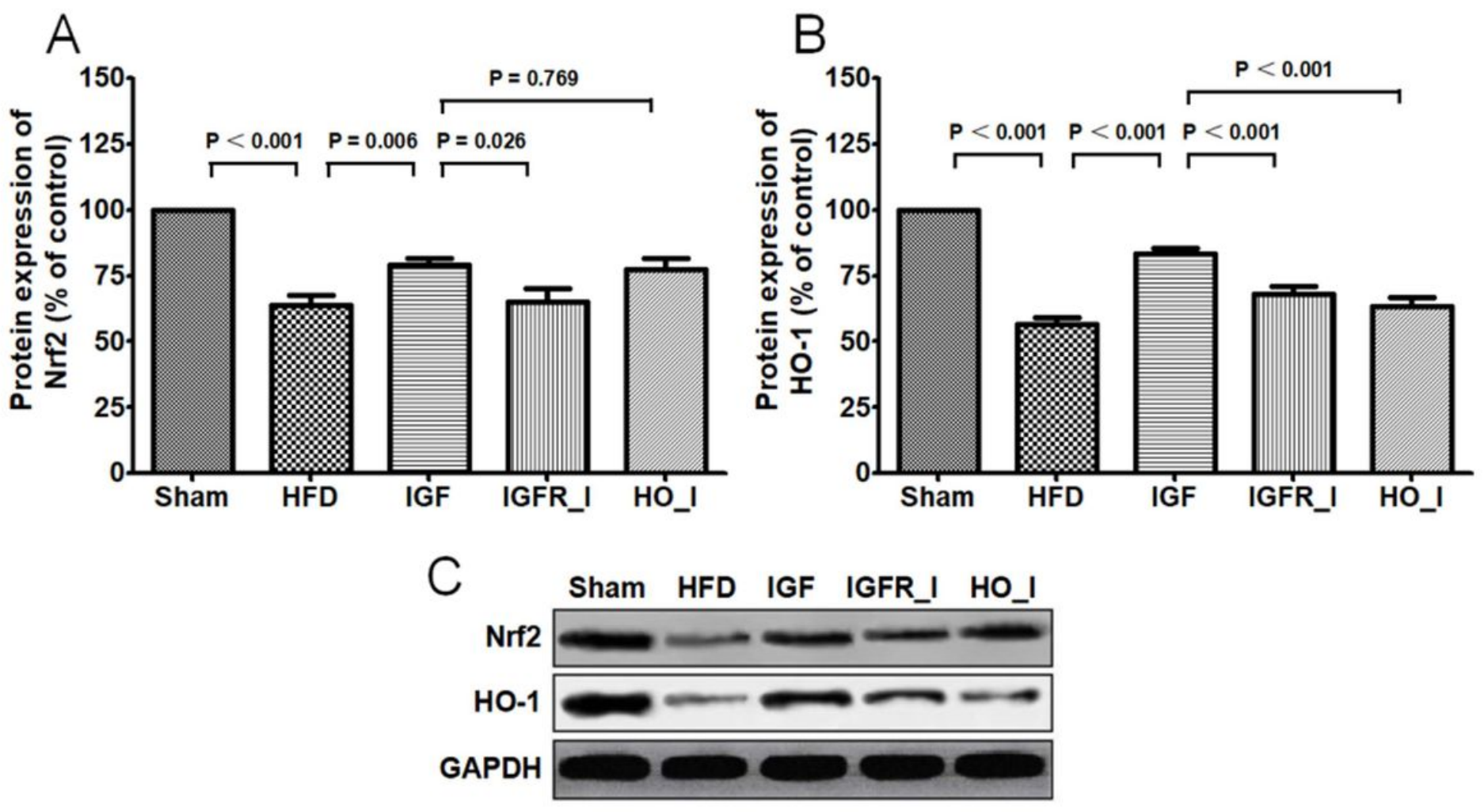

Figure 3

Expression of Nrf2 and HO-1 in animal groups The hippocampus tissue specimens were obtained. Expression of Nrf2 and HO-1 was determined using western blotting Variable comparison between different groups was performed using ANOVA with LSD test. $P \otimes 0.05$ was regarded as statistically significant.
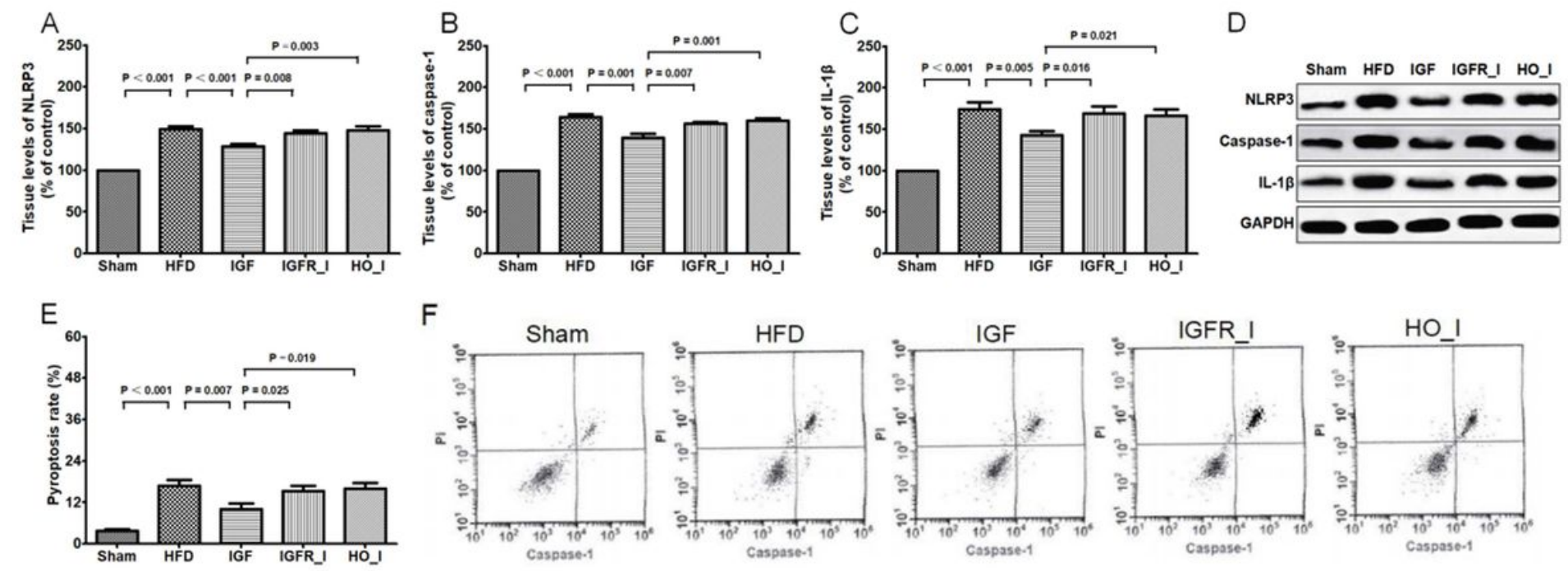

Figure 4

Pyroptosis in animal groups Expression of NLRP3, Caspase-1 and IL-1 $\beta$ in the hippocampus was determined using western blotting. Pyroptosis rate was measured using flow cytometry. Variable 
comparison between different groups was performed using ANOVA with LSD test. P $\otimes 0.05$ was regarded as statistically significant.
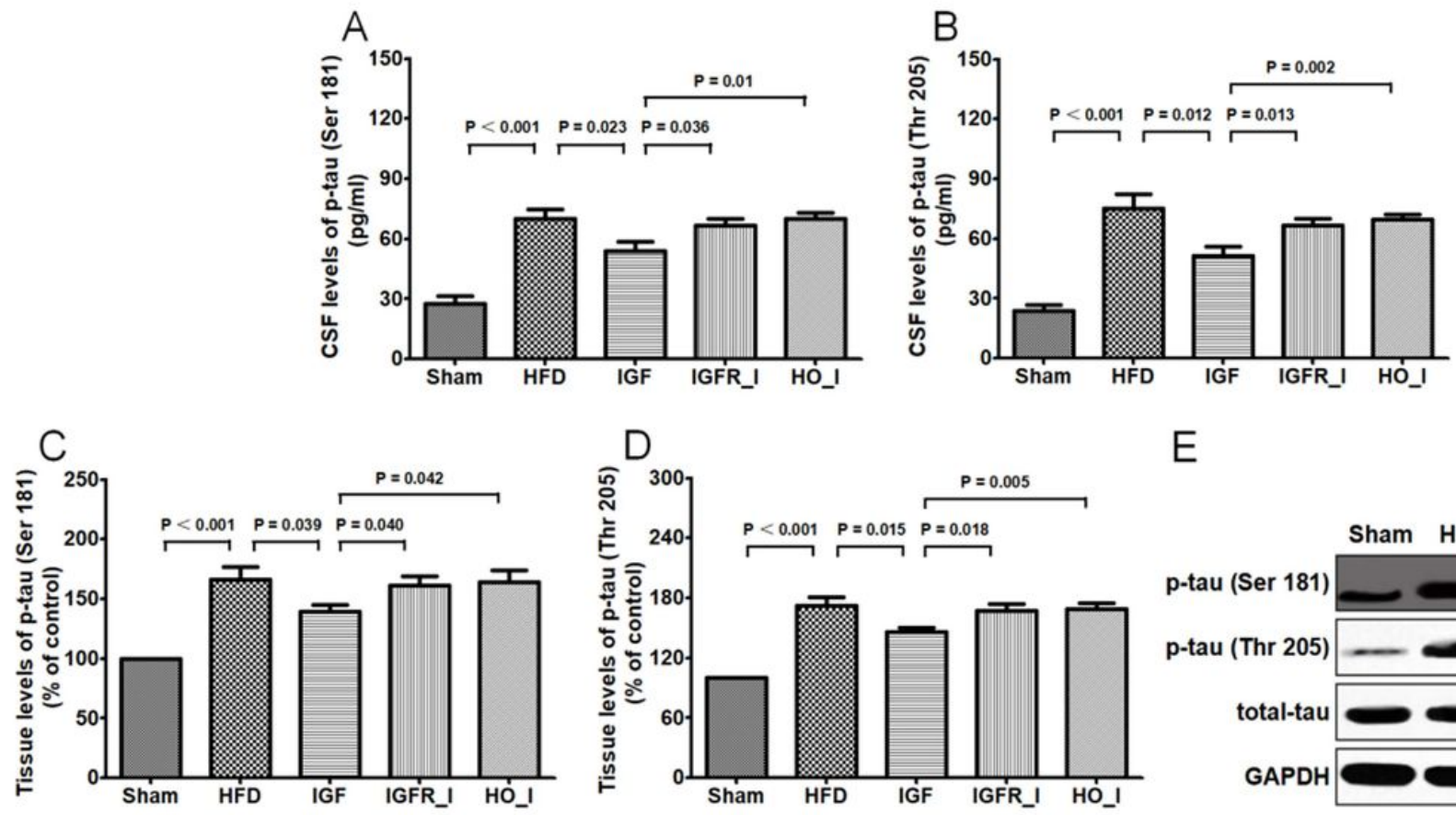

$E$

\section{Figure 5}

Expression of tau proteins in animal groups Cerebrospinal fluid levels of p-tau (Ser 181) and p-tau (Thr 205) were determined using a Singulex Erenna immunoassay platform. Expression of p-tau (Ser 181), ptau (Thr 205) and total-tau in the hippocampus was determined using western blotting. Variable comparison between different groups was performed using ANOVA with LSD test. P $\otimes 0.05$ was regarded as statistically significant.
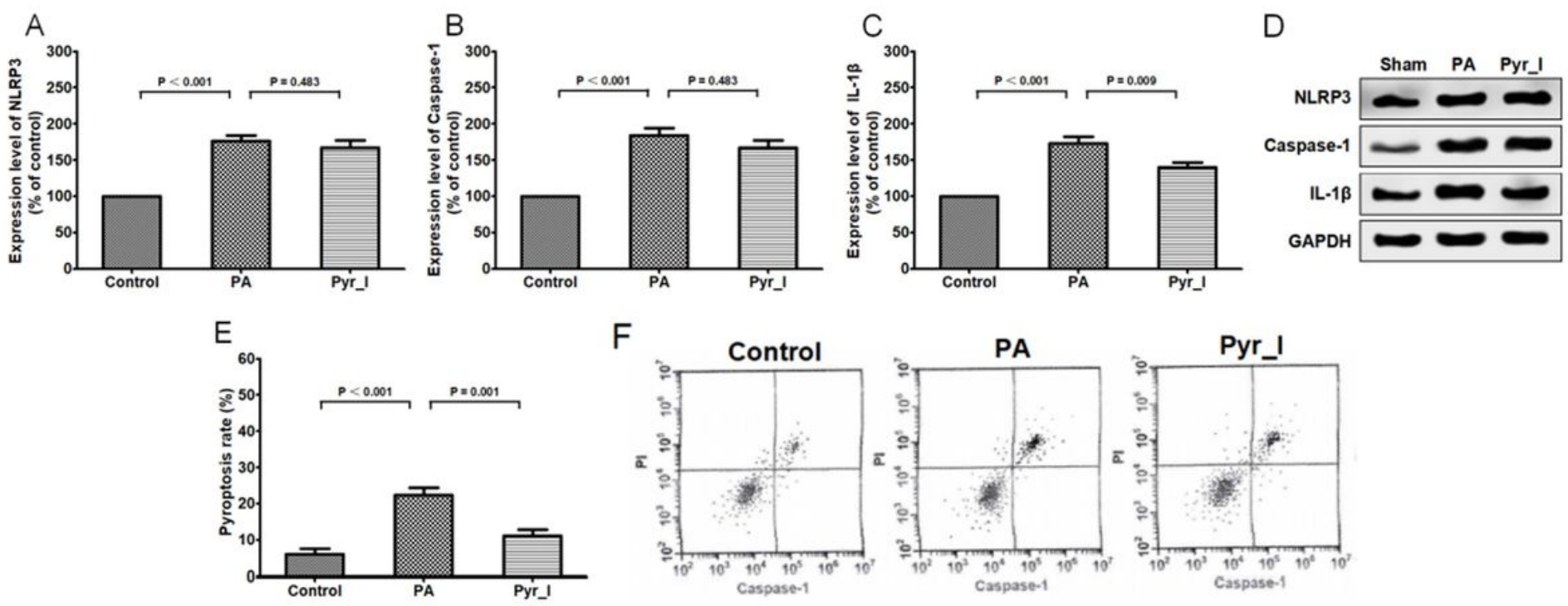

Figure 6 
Pyroptosis in hippocampal neuron groups The hippocampal neurons were randomly divided into three groups: control group, palmic acid treatment group (PA group) and pyroptosis inhibition group (Pyr_I group). Each group contained ten wells. The cells in the PA and Pyr_I groups were treated with palmic acid, and the cells in the Pyr_l group were also treated with necrosulfonamide. Expression of NLRP3, Caspase- 1 and IL-1 $\beta$ in the neurons were determined using western blotting. Pyroptosis rate was measured using flow cytometry. Variable comparison between different groups was performed using ANOVA with LSD test. P $\otimes 0.05$ was regarded as statistically significant.
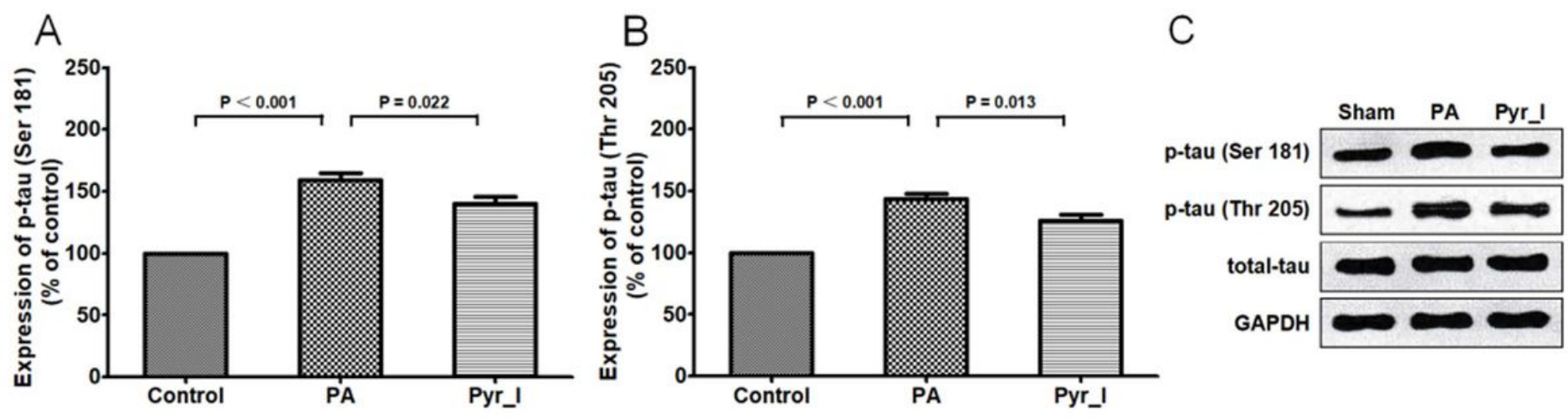

\section{Figure 7}

Expression of tau proteins in hippocampal neuron groups Expression of p-tau (Ser 181), p-tau (Thr 205) and total-tau in the hippocampal neurons was determined using western blotting. Variable comparison between different groups was performed using ANOVA with LSD test. $\mathrm{P} \otimes 0.05$ was regarded as statistically significant. 